\title{
Computing Realistic Surrogate EEG for the Study of Functional Connectivity
}

\author{
Christian O'Reilly \\ Montreal Neurological Institute-Hospital, \\ Azrieli Centre for Autism Research \\ McGill University \\ Montreal, Canada \\ christian.oreilly@mcgill.ca
}

\author{
Mayada Elsabbagh \\ Montreal Neurological Institute-Hospital, \\ Azrieli Centre for Autism Research \\ McGill University \\ Montreal, Canada \\ mayada.elsabbagh@mcgill.ca
}

\begin{abstract}
Functional connectivity computed from electroencephalograms (EEG) can be used to better understand how the brain works. Unfortunately, estimating such connectivity is fraught with many pitfalls and can be confounded with artifacts due to volume conduction, common sources, reference scheme, etc. Devising a method to compute surrogate EEG that would be free of functional connectivity but that would reliably reproduce the effect of confounders such as volume conduction would be invaluable for statistical inference on functional connectivity. We developed such a method by simulating EEG from estimated sources and by reproducing the properties of local (but not long-range) functional connectivity in intracranial recordings. We present an example of how this approach can be used to improve the estimation of functional connectivity in EEG.
\end{abstract}

Keywords-EEG; volume conduction; surrogate; zero-lag synchrony; functional connectivity

\section{INTRODUCTION}

The electroencephalogram (EEG) records neural activity using scalp electrodes. For this to be possible, the electric field generated by the neuronal sources has to propagate through the different tissues of the head to reach the scalp, a process referred to as volume conduction. At the frequencies relevant for EEG (typically $<100 \mathrm{~Hz}$ ), the reactance of head tissues is negligible such that volume conduction is essentially instantaneous [1]. When comparing the activity picked up by pairs of EEG electrodes to determine their statistical dependence (i.e., functional connectivity), any interdependence can be due to a mixture of 1) volume conducted activity being propagated simultaneously to both electrodes and 2) neuronal activity being communicated between brain regions through bundles of long-range axons relaying action potentials. The first component is normally considered to be a confounder of no interest while the second is the phenomenon of interest when studying functional connectivity between brain regions. To estimate the effect of the latter without being biased by the former, the common approach is to reject any instantaneous (unlagged) synchronization between pairs of channels [2]. However, it is well understood that although neural networks are linked through delayed connections (i.e., synaptic transmission and propagation of action potentials along axons are not instantaneous), they can nevertheless synchronize with no lag due to reciprocal interactions between brain regions [3]. To demonstrate the existence and importance of zero-lag connectivity, we previously used intracranial recordings which are known to be susceptible to volume conduction only up to around 20 $\mathrm{mm}$ [4]. In doing so, we demonstrated robust and ubiquitous zero-lag connectivity between long-range (>100 mm) homotopic brain regions [5].

Since intracranial recordings and EEG are sensitive to patterns of synchrony at different spatial scales, it is yet unclear to what extent zero-lag connectivity is important in EEG. Gaining more clarity on this topic is not trivial since zero-lag synchrony is markedly more difficult to study in EEG than in intracranial recordings because volume conduction can propagate at least up to $100 \mathrm{~mm}$ in EEG [4]. A reliable method to obtain surrogate EEG that faithfully captures the effect of volume conduction without containing any functional connectivity would be invaluable for this study. For example, surrogate EEG could be used to remove the contribution of volume conduction from estimated connectivity. In this communication, we propose an approach to computing such realistic surrogate EEG.

\section{MethodS}

\section{A. Datasets}

We used two open-access datasets in this study. The first one contains intracranial recordings from 106 patients (54 males; mean age of $33.1 \pm 10.8$ years) who were candidates for surgical treatment of drug-resistant epilepsy [6]. Only channels from brain regions considered "very likely to be healthy" by a consensus of two epileptologists we included. Recordings were sampled at $200 \mathrm{~Hz}$ and filtered to the $0.5-80 \mathrm{~Hz}$ band. Sixty seconds of artifact-free recording is available for each recording channel (1772 in total) in wakefulness and sleep. We used only the recording in wakefulness.

The second dataset (MPI-LEMON) contains multimodal recordings (magnetic resonance imaging (MRI), EEG, cognition, emotion, peripheral physiology) in young $(\mathrm{N}=153,25.1 \pm 3.1$ years, range $20-35$ years, 45 females) and elderly $(\mathrm{N}=74,67.6 \pm 4.7$ years, range 59-77 years, 37 females) healthy adults. We only used the 10 first epochs (for computational reasons) of eyes-open resting-state EEG for the subset of 82 
subjects who had structural MRI (T1w and FLAIR), digitized electrode positions, and EEG sampled at 250 Hz. EEG was recorded with a BrainAmp MR plus amplifier and an active ActiCAP 62-channel net (Brain Products $\mathrm{GmbH}$, Gilching, Germany) following the 10-10 system and referenced to FCz. Electrode positions were digitized using a Polhemus PATRIOT system (Polhemus, Colchester, VT, USA) [7].

\section{B. Source estimation and EEG simulation}

We used the following approach to compute realistic surrogate EEG that captures the dynamics of volume propagation but contains no functional connectivity: 1) estimate the EEG cortical sources, 2) remove any functional connectivity between these sources, and 3) simulate the scalp EEG that such source distribution would generate. For this process, we used the fsaverage head model, as available in MNE-Python. Although the MPI-LEMON dataset includes structural MRI which can be used to compute individual subject head models, we used the fsaverage template to derive a solution that does not depend on subject-specific MRI and is therefore usable in any EEG studies with sufficient sensor coverage to support proper source reconstruction. We first co-registered the subject's electrode montages to this head model (Fig. 1.a) and we fixed the orientation of the dipolar sources perpendicular to the cortical mesh. Then, we computed the source activations using a diagonal noise matrix and the eLORETA inverse operator. a

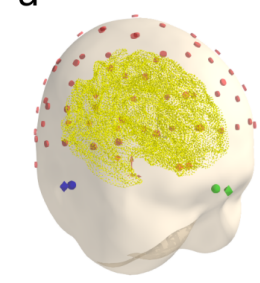

b

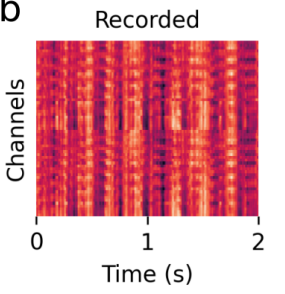

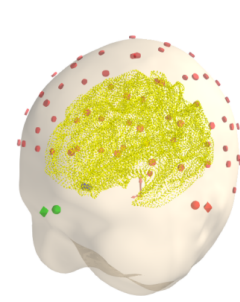

Simulated

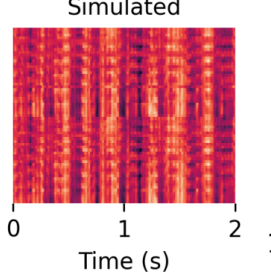

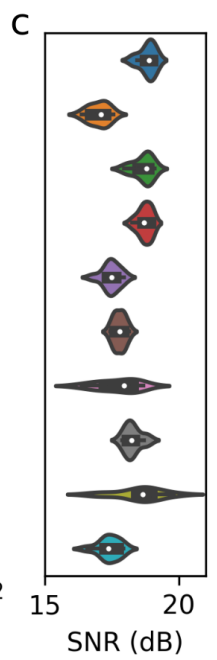

Fig. 1. Validation of the source estimation and EEG simulation process. a) Co-registration of the EEG channels with the fsaverage head model. b) Comparison between a recorded EEG epoch and the EEG simulated from the corresponding cortical sources $(\mathrm{SNR}=18.4$ dB). c) Quality of the simulation (SNR) across epochs for 10 typical subjects.

Using the same head model, we can simulate the EEG that such a source distribution would produce and verify that it reproduces accurately the recorded EEG (Fig 1.b). The degree to which the simulation successfully reproduce the original EEG can be assessed by computing the following signal-to-noise ratio (SNR)

$$
S N R=10 \log _{10}\left(\frac{\overline{|x+y|}}{2 \overline{|x-y|}}\right)
$$

with $\mathrm{x}$ and $\mathrm{y}$ being respectively the recorded and the simulated EEG for a given epoch. The example shown in Fig 1.b has a $18.4 \mathrm{~dB}$ SNR and it is representative of the quality obtained across recordings (Fig 1.c).

\section{Functional connectivity}

We used the absolute value of the coherency (hereafter just referred to as coherence) to estimate the functional connectivity between pairs of channels

$$
C(f)=\left|\frac{\left.E\left[S_{X Y}(f)\right)\right]}{\sqrt{\left.\left.E\left[S_{X X}(f)\right)\right] E\left[S_{Y Y}(f)\right)\right]}}\right|
$$

where $E[\ldots]$ indicates the average across epochs and $S_{x y}$ stands for the cross-spectral density.

\section{Surrogate computation}

Surrogate EEG can be computed using the same process used to simulate EEG from estimated cortical sources, but removing functional connectivity between sources before using them for simulation. Depending on the objectives pursued by a given analysis, different approaches can be considered. We compared three alternatives: 1) spatial randomization, 2) phase randomization, and 3 ) phase randomization corrected to account for local synchrony. The first approach simply randomizes the position of the sources. It has the advantage of being conceptually simple and of not altering in the source time-series themselves, only their spatial relationship to one another. This approach, therefore, destroys any systematic spatial pattern of functional connectivity, allowing the comparison of surrogate data with recorded EEG (e.g., using cluster-based permutation tests) to assess if the topology of functional connectivity is statistically significant. It does not, however, remove the functional connectivity between individual pairs of sources.

In the second approach (phase randomization), the Fast Fourier Transform (FFT) of the source time-series is computed, its phase is randomized, and the inverse FFT of the phase-randomized spectrum is computed to obtain signals that have the same spectral power as the original signals, but which phase has been scrambled. In doing so, we preserve the spectral power of the sources, but we eliminate any phase dependency (i.e., functional connectivity) between these sources.

The third approach is based on the second but further compensates for the fact that local cell assemblies need to be synchronized (i.e., functionally connected) for scalp EEG to be produced. It is nonsensical to consider EEG generated by unsynchronized neuronal populations because for EEG to have sufficient amplitude to travel up to the scalp, it must be produced by locally synchronized neuronal activity. An ideal surrogate dataset would simulate the properties of EEG sources as they were before being smeared by volume conduction (i.e., spatially extended sources produced by sufficient local synchrony), but devoid of long-range functional connectivity. To reproduce the properties of the EEG cortical sources before they get smeared by volume conduction, we turned to intracranial recordings which capture local field potentials (LFP) in the brain. We modeled how the 
Preprint submitted and accepted for publication in the proceedings of the

IEEE-EMBS International Conference on Biomedical and Health Informatics 2021.

Published version will be available on IEEE Xplore.

absolute coherency decreases with distance $(d)$ between intracranial electrodes by fitting a decreasing exponential function $\sigma(d)$ (see Fig. 2).

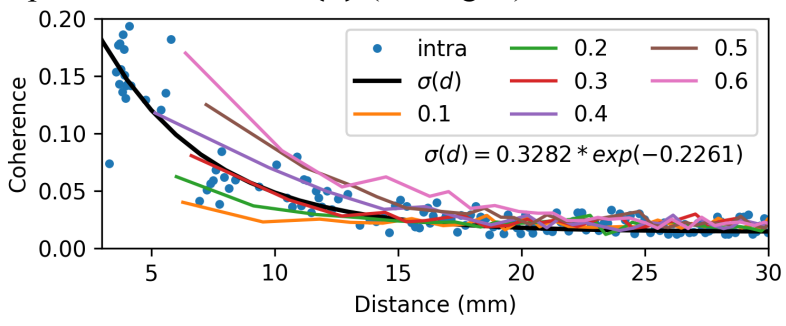

Fig. 2. Adjusting the EEG sources to reproduce the level of coherence found in LFP. We fitted a decreasing exponential (black line; equation below the legend) to the coherence computed between implanted electrodes. The coherence has been computed between every pair of implanted electrodes, within every subject. These values have been sorted by inter-electrode distance and average by bins of 50 pairs (blue points). We tested different $c$ values, from 0.1 to 0.6 .

To correlate the phase-randomized sources so that their local synchrony reproduces the relationship captured by $\sigma(d)$, we used a distance threshold $T=20 \mathrm{~mm}$ to select sources that are in a range susceptible to local coherence (see Fig 2). For a source $s_{i}$, we used a k-d tree [8] to find the set of neighbor sources, defined as the sources $s_{j}$ such that $d\left(s_{i}, s_{j}\right)<T$ with $d\left(s_{i}, s_{j}\right)$ standing for the Euclidean distance between $s_{i}$ and $s_{j}$. Then, we randomly selected a sub-sample $\Theta_{i}$ containing $c \%$ of these neighbor sources. This step models the fact that within a local region, not all neurons are tightly correlated in the brain and it allows for patterns of activity with higher spatial frequencies. Further, correlating all local sources results in local coherence larger than what is observed in LFP (see Fig. 2). The corrected source $\widehat{s_{i}}$ is obtained as a weighted sum of the original source $s_{i}$ and its selected set of neighbor sources $s_{j} \in \Theta_{i}$, using $\sigma(d)$ as a weighting function

$$
\begin{gathered}
\widetilde{s_{i}}=s_{i}+\sum_{s_{j} \in \Theta_{i}} \sigma\left(d\left(s_{i}, s_{j}\right)\right) s_{j} \\
\widehat{s_{i}}=\widetilde{s_{i}} \frac{\overline{\left|\widetilde{s_{i}}\right|}}{\mid \widetilde{s_{i} \mid}}
\end{gathered}
$$

with (3.b) used to normalize the sources computed in (3.a) so that $s_{i}$ and $\widehat{s_{i}}$ have the same average amplitude. We tested different values for the proportion $c$ (Fig 2) and selected $c=0.35$ as a good approximate.

We further note that the sources $\widehat{s}_{i}$ are less locally correlated than the sources estimated from EEG. This suggests that common approaches for estimating EEG sources overestimate the local source correlation. It also means that our surrogate EEG will have a smaller amplitude than the recorded EEG since the contributions of locally uncorrelated sources do not sum constructively to generate high amplitude electrical fields. To compensate for this effect, we take advantage of the fact that the sources and the simulated EEG are associated through a linear relationship. Thus, the amplitude of the sources $\widehat{S}_{i}$ and the surrogate EEG can be adjusted by scaling them by the ratio between the amplitude of the recorded and the surrogate EEG.

\section{RESULTS}

We compared the coherence obtained with an average reference and the scalp current density (SCD) [9] within (wtn) and between (btwn) subjects (Fig 3., left panel). The SCD provides reference-free estimates of neuronal activity. Compared to the average reference, it is more sensitive to higher spatial frequencies and less impacted by volume conduction [10].
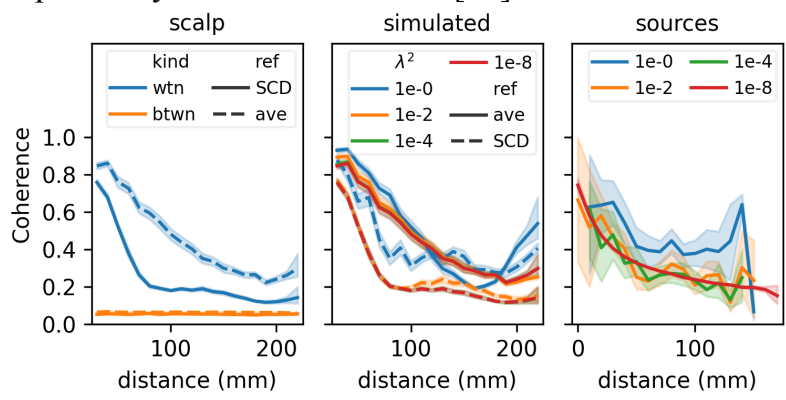

Fig. 3. Impact of using the average reference (ave) or SCD to compute the coherence between channels or sources. The left panel also compares coherence within-subject (wtn) and between-subject (btwn). The middle and right panels further compare the effect of different values of $\lambda^{2}$ on the sources and the estimated EEG. Shaded regions indicate the bootstrapped $95 \%$ interval of confidence.

The between-subject comparison is a sanity check since no functional connectivity is expected to be present when comparing EEG signals between subjects. We observed a small but nonnull average coherence between subjects, as expected for any noisy process bound to the $[0,1]$ interval. This bias can be removed by subtracting the coherence from a surrogate dataset as we do below (see Fig. 5).

We then estimated the cortical sources using different values for the regularization parameter $\lambda^{2}$ and simulated EEG from these sources (Fig. 3, middle and right panels). Since the regularization parameter $\lambda^{2}$ spatially smooths the sources, it is expected to have a detrimental impact on the spatial resolution of the sources. As shown in Fig. 3, large values of $\lambda^{2}$ can have an impact on our ability to reproduce the original EEG from the sources as it introduces too much coherence between the sources, which is reflected in turn in the estimated EEG. For the following analyses, we used $\lambda^{2}=10^{-4}$ as it appears to be sufficiently small to estimate sources that can accurately reproduce the original EEG. SNRs in Fig. 1 were also obtained from simulations using this $\lambda^{2}$ value.

We then compared the coherence from the average reference EEG, SCD, and cortical sources using the recorded EEG (rec) and the three surrogate approaches previously described: spatial randomization (spa), phase randomization (pha), and phase randomization corrected to account for local synchrony (cor) (Fig. 4). We also added to the source plot the coherence for the intracranial recordings (intra). An inset added to the right panel of Fig. 4 shows that the coherence computed using the corrected surrogate with $\mathrm{c}=0.35$ closely follows what was found in LFP. 


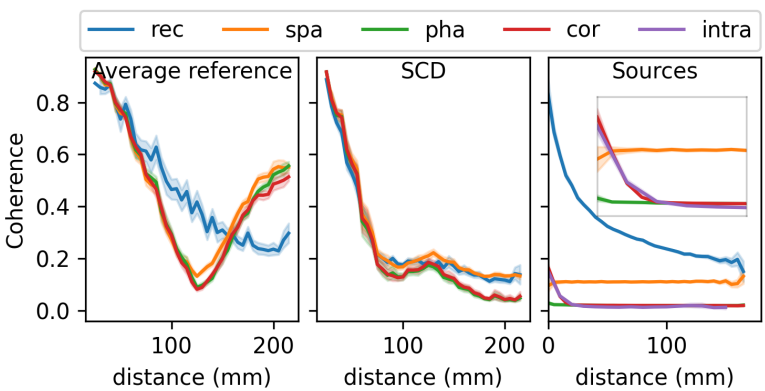

Fig. 4. Comparison of the coherence computed on the recorded EEG (rec), three types of EEG surrogates (spa: spatial randomization; pha: phase randomization; cor: corrected phase randomization), and intracranial recordings (intra). Shaded regions indicate the bootstrapped $95 \%$ interval of confidence.

Using the corrected phase-randomized surrogate dataset, we computed the 95th percentile of the coherence distribution per channel pair and subtracted it to the coherence estimated on the recorded EEG so that any remaining positive coherence would indicate statistical significance (before any correction is applied for multiple tests) (Fig. 5).

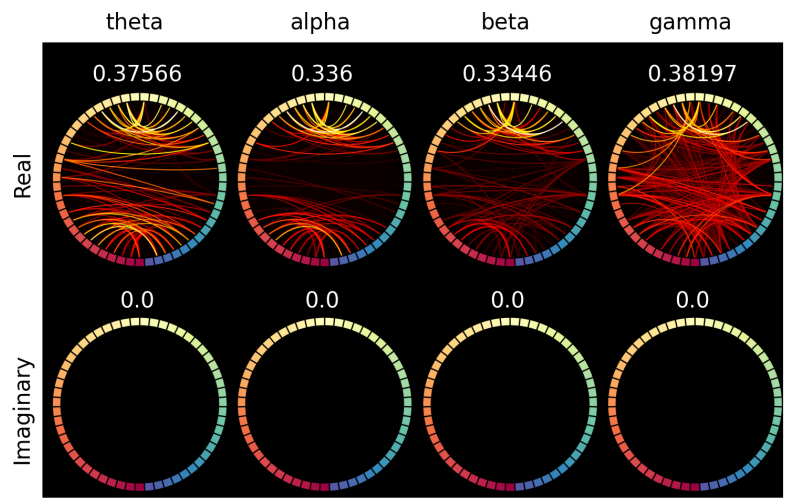

Fig. 5. Circular connectivity plot for the real (top) and imaginary (bottom) part of coherency computed on SCD of recorded EEG after removing the 95th percentile of the corrected phase-randomized surrogate distribution. The $\mathrm{Oz}$ channel is placed at the bottom of these circles. Turning clockwise, the channels from the left side of the head are positioned by increasing anteroposterior coordinate. The right side channels are positioned correspondingly on the other half of the circle. The connections are color-coded with a "hot" colormap, going from black (0) to white (value shown at the top of each circular graph). All midline channels except $\mathrm{Oz}$ (i.e., $\mathrm{Fz}, \mathrm{Cz}, \mathrm{AFz}, \mathrm{Pz}, \mathrm{CPz}, \mathrm{POz}$ ) were rejected to avoid creating left-right asymmetry in the graphs.

\section{Discussion}

In this paper, we developed and compared different ways to compute surrogate EEG to untangle volume conduction from functional connectivity without systematically discarding zero-lag synchrony. This approach can be deployed to investigate the property of zero-lag connectivity in the brain. In a previous study, we showed using intracranial recordings that the most significant long-range functional connectivity in LFP is happening at zero-lag between homotopic brain regions [5]. It is therefore worth noting the predominance of interhemispheric connectivity in the theta band (Fig. 5) for the real component of the coherency (i.e., the component that is sensitive to zero-lag synchrony) after correcting for the potential confounding effect of volume conduction.
Further, comparing the real (sensitive to zero-lag synchrony) and the imaginary (not sensitive to zero-lag synchrony) part of coherency suggests that neuronal activity in the brain is likely to be dominated by zero and near-zero lag synchrony. Actually, the imaginary part of coherency did not survive the correction using the surrogate dataset for any connections.

Although a proper demonstration of the statistical significance of these observations and the detailed study of zero-lag connectivity is out of the scope of this short communication, these results are coherent with what we observed in more detailed analyses (manuscript being prepared for publication). By describing this surrogate approach we hope to motivate members of the neuroimaging community to improve their methods to estimate functional connectivity so that they deal with volume conduction in a more nuanced way and provide a more faithful description of the functional connectivity in the brain that includes any physiological zero-lag synchrony.

The code written for these analyses is available on GitHub (https://github.com/christian-oreilly).

\section{ACKNOWLEDGMENT}

This research is supported by the Azrieli Centre for Autism Research (ACAR) and the Fonds de recherche du Québec - Santé (FRQS).

\section{REFERENCES}

[1] R. Plonsey and D. B. Heppner, "Considerations of quasi-stationarity in electrophysiological systems," Bull. Math. Biophys., vol. 29, no. 4, pp. 657-664, Dec. 1967, doi: 10.1007/BF02476917.

[2] G. Nolte, O. Bai, L. Wheaton, Z. Mari, S. Vorbach, and M. Hallett, "Identifying true brain interaction from EEG data using the imaginary part of coherency," Clin. Neurophysiol. Off. J. Int. Fed. Clin. Neurophysiol., vol. 115, no. 10, pp. 2292-2307, Oct. 2004, doi: 10.1016/j.clinph.2004.04.029.

[3] R. Vicente, L. L. Gollo, C. R. Mirasso, I. Fischer, and G. Pipa, "Dynamical relaying can yield zero time lag neuronal synchrony despite long conduction delays," Proc. Natl. Acad. Sci. U. S. A., vol. 105, no. 44, pp. 17157-17162, Nov. 2008, doi: 10.1073/pnas.0809353105.

[4] P. L. Nunez et al., "EEG coherency. I: Statistics, reference electrode, volume conduction, Laplacians, cortical imaging, and interpretation at multiple scales," Electroencephalogr. Clin. Neurophysiol., vol. 103, no. 5, pp. 499-515, Nov. 1997, doi: 10.1016/s0013-4694(97)00066-7.

[5] C. O'Reilly and M. Elsabbagh, "Intracranial recordings reveal ubiquitous in-phase and in-antiphase functional connectivity between homotopic brain regions in humans," J. Neurosci. Res., Nov. 2020, doi: 10.1002/jnr.24748.

[6] B. Frauscher et al., "Atlas of the normal intracranial electroencephalogram: neurophysiological awake activity in different cortical areas," Brain, vol. 141, no. 4, pp. 1130-1144, Apr. 2018, doi: 10.1093/brain/awy035.

[7] A. Babayan et al., "A mind-brain-body dataset of MRI, EEG, cognition, emotion, and peripheral physiology in young and old adults," Sci. Data, vol. 6, no. 1, Art. no. 1, Feb. 2019, doi: 10.1038/sdata.2018.308.

[8] J. L. Bentley, "Multidimensional binary search trees used for associative searching," Commun. ACM, vol. 18, no. 9, pp. 509-517, Sep. 1975, doi: 10.1145/361002.361007.

[9] F. Perrin, O. Bertrand, and J. Pernier, "Scalp Current Density Mapping: Value and Estimation from Potential Data," IEEE Trans. Biomed. Eng., vol. BME-34, no. 4, pp. 283-288, Apr. 1987, doi: 10.1109/TBME.1987.326089.

[10] P. L. Nunez and R. Srinivasan, Electric Fields of the Brain: The neurophysics of EEG, 2nd ed. Oxford; New York: Oxford University Press, 2006. 